\title{
BMJ Open Strategies to identify individuals with monogenic diabetes: results of an economic evaluation
}

\author{
Jaime L Peters (10 , ${ }^{1,2}$ Rob Anderson, ${ }^{3}$ Beverley Shields, ${ }^{4}$ Sophie King, ${ }^{4}$ \\ Michelle Hudson (D) , ${ }^{4}$ Maggie Shepherd (D) , ${ }^{4}$ Timothy James McDonald, ${ }^{4,5}$ \\ Ewan Pearson, ${ }^{6}$ Andrew Hattersley, ${ }^{4}$ Chris Hyde ${ }^{1}$
}

To cite: Peters JL, Anderson R, Shields B, et al. Strategies to identify individuals with monogenic diabetes: results of an economic evaluation. BMJ Open 2020;10:e034716. doi:10.1136/ bmjopen-2019-034716

- Prepublication history and additional material for this paper are available online. To view these files, please visit the journal online (http://dx.doi. org/10.1136/bmjopen-2019034716).

Received 02 October 2019 Revised 09 January 2020 Accepted 30 January 2020

\section{Check for updates}

(c) Author(s) (or their employer(s)) 2020. Re-use permitted under CC BY. Published by BMJ.

${ }^{1}$ Exeter Test Group, University of Exeter Medical School, Exeter, UK

${ }^{2}$ Collaboration for Leadership in Applied Health Research and Care South West Peninsula (NIHR CLAHRC South West Peninsula), University of Exeter Medical School, Exeter, UK ${ }^{3}$ ESMI (Evidence Synthesis \& Modelling for Health Improvement), University of Exeter, Exeter, UK

${ }^{4}$ NIHR Clinical Research Facility, University of Exeter Medical School, Exeter, UK

${ }^{5}$ Royal Devon and Exeter NHS Foundation Trust, Exeter, UK

${ }^{6}$ Division of Molecular \& Clinical Medicine, University of Dundee, Dundee, UK

Correspondence to

Dr Jaime L Peters;

j.peters@exeter.ac.uk

\section{ABSTRACT}

Objectives To evaluate and compare the lifetime costs associated with strategies to identify individuals with monogenic diabetes and change their treatment to more appropriate therapy.

Design A decision analytical model from the perspective of the National Health Service (NHS) in England and Wales was developed and analysed. The model was informed by the literature, routinely collected data and a clinical study conducted in parallel with the modelling.

Setting Secondary care in the UK.

Participants Simulations based on characteristics of patients diagnosed with diabetes $<30$ years old.

Interventions Four test-treatment strategies to identify individuals with monogenic diabetes in a prevalent cohort of diabetics diagnosed under the age of 30 years were modelled: clinician-based genetic test referral, targeted genetic testing based on clinical prediction models, targeted genetic testing based on biomarkers, and blanket genetic testing. The results of the test-treatment strategies were compared with a strategy of no genetic testing.

Primary and secondary outcome measures Discounted lifetime costs, proportion of cases of monogenic diabetes identified.

Results Based on current evidence, strategies using clinical characteristics or biomarkers were estimated to save approximately £100-£200 per person with diabetes over a lifetime compared with no testing. Sensitivity analyses indicated that the prevalence of monogenic diabetes, the uptake of testing, and the frequency of home blood glucose monitoring had the largest impact on the results (ranging from savings of $£ 400-£ 50$ per person), but did not change the overall findings. The model is limited by many model inputs being based on very few individuals, and some long-term data informed by clinical opinion.

Conclusions Costs to the NHS could be saved with targeted genetic testing based on clinical characteristics or biomarkers. More research should focus on the economic case for the use of such strategies closer to the time of diabetes diagnosis.

Trial registration number NCT01238380.

\section{BACKGROUND}

Monogenic diabetes is a form of diabetes caused by a mutation in a single gene, which
Strengths and limitations of this study

- The model structure was informed by expert consultation and critical appraisal of existing models.

- Parameter values were taken from a UK-based clinical study conducted alongside this economic evaluation.

Wide-ranging sensitivity analyses were conducted.

- Many parameters were based on low numbers of patients

Evidence on effectiveness was limited.

is inherited in an autosomal dominant manner. ${ }^{1}$ Therefore a child of an individual with monogenic diabetes has a $50 \%$ chance of inheriting the mutation (assuming the child's other parent does not have the mutation). Mutations in glucokinase $(G C K)$, hepatocyte nuclear factor 1 alpha $(H N F 1 A)$ and hepatocyte nuclear factor 4 alpha $(H N F 4 A)$ genes are the most common forms of monogenic diabetes. ${ }^{2}$ Individuals with mutations in the GCK gene have persistently moderately raised blood glucose levels from birth, that is rarely detrimental to health ${ }^{3}$ and does not respond to treatment. ${ }^{4}$ Therefore individuals with mutations in the $G C K$ gene can be successfully treated by diet. ${ }^{4}$ Individuals with $H N F 1 A$ or HNF4A mutations have blood glucose levels which increase over time and can be successfully treated with sulphonylureas ${ }^{5}$ but may, eventually, require insulin treatment. ${ }^{6}$

The minimum prevalence of monogenic diabetes in the UK has been estimated as 108 cases per million. ${ }^{7}$ As it usually presents by 25-30 years of age, ${ }^{128}$ individuals are often misdiagnosed with type 1 diabetes, and receive insulin treatment when less invasive and less costly treatment is more appropriate.

The National Health Service (NHS) in England and Wales currently has no national guidelines for identifying individuals with monogenic diabetes. Realistic strategies are 
available ranging from genetic testing of all individuals with diabetes to targeted genetic testing based on clinical characteristics $^{9}$ or biochemical $^{10}$ and immunological ${ }^{11}$ tests. We report a UK-based economic evaluation of these realistic strategies to identify individuals with monogenic diabetes (defined here as mutations in GCK, HNF1A or HNF4A genes). The development of the model-based economic evaluation has been published elsewhere. ${ }^{12}$ The economic evaluation was undertaken alongside a clinical study whose aims included (1) Investigating the prevalence of monogenic diabetes within two areas of the UK. (2) Measuring the effects of a change of treatment following a positive diagnosis of monogenic diabetes. The clinical study recruited 1407 individuals who were diagnosed with diabetes $<30$ years old and who were $<50$ years old at recruitment. ${ }^{13}$ Prospective quality of life using the EuroQol (EQ-5D) Index, a generic measure of health outcome $^{14}$ and glycated haemoglobin (HbAlc) data for 45 individuals who were diagnosed with monogenic diabetes within the geographical areas of the clinical study were collected until 12 months after the genetic test result. Although the clinical study collected data on clinical outcomes, it was not designed, nor powered, to detect small changes in clinical outcomes. No statistically significant change in the EQ-5D Index or HbA1c before and 12 months after changing treatment was observed making it impossible to confirm or refute the clinically suspected benefit of changing treatment in persons found to have monogenic diabetes, but on inappropriate treatment. Thus, for the main analyses, only costs are considered in this economic evaluation, making this a conservative analysis of the testing strategies if patient benefit does occur. The implications of this are considered in the discussion.

The aim of this analysis is to evaluate and compare the lifetime costs of different realistic strategies in the NHS to identify individuals with monogenic diabetes and change their treatment to more appropriate therapy. This economic evaluation has been reported in line with the Consolidated Health Economic Evaluation Reporting Standards. ${ }^{15}$

\section{MATERIALS AND METHODS \\ Model overview}

A hybrid decision model was developed from the perspective of the NHS in England and Wales. A decision tree was developed in MicroSoft Excel to estimate the shortterm (16 months) costs, which allowed a maximum of 4 months from referral to testing to change of treatment (for those identified as having monogenic diabetes), plus 12 months follow-up (coinciding with the accompanying clinical study). The IMS Centre for Outcomes Research (CORE) Diabetes Model (IMS CDM) V.8.5 ${ }^{16}$ was used to estimate the lifetime costs associated with the strategies. Expert consultation and explicit critical appraisal of existing long-term diabetes models helped to inform the structure of the decision model and choice of the IMS CDM (see Peters et $a l^{12}$ for more detail on model

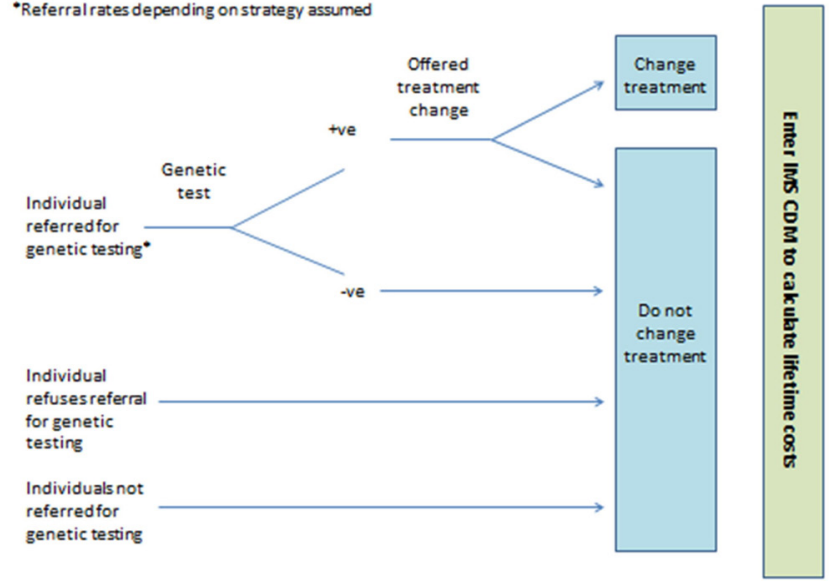

Figure 1 Simplified model structure for the Ad Hoc Testing, Clinical Prediction Model Testing and All Testing strategies. IMS CDM, IMS CORE Diabetes Model.

development). Evidence to inform the model came from a number of sources including published and unpublished data and clinical opinion. Details on the evidence used in the model are given below.

\section{Strategies and comparator}

Five strategies for identifying monogenic diabetes in individuals who were diagnosed with diabetes under the age of 30 years were defined: no genetic testing ('No Testing'), clinician-based genetic test referral ('Ad Hoc Testing'), targeted genetic testing based on clinical prediction models ${ }^{9}$ ('Clinical Prediction Model Testing') or biochemical (urinary $\mathrm{C}$ peptide to creatinine ratio, $\mathrm{UCPCR})^{10}$ and immunological (islet autoantibodies) ${ }^{11}$ test results ('Biomarker Testing'), blanket genetic testing ('All Testing').

The No Testing strategy is the comparator for all other strategies, as it represents the current policy within England and Wales where there is no guidance on the identification of individuals with monogenic diabetes. Thus, in this strategy all individuals remain on the diabetes treatment they were receiving at the start of the model, regardless of whether they truly have monogenic diabetes or not.

The Ad Hoc Testing strategy assumes no systematic referral of individuals for monogenic diabetes genetic testing. Instead, individuals are referred on an ad hoc basis depending on the awareness of local clinicians of monogenic diabetes (see figure 1). Data on referral rates for monogenic diabetes genetic testing in the $\mathrm{UK}^{7}$ were used to calculate estimates of sensitivity and specificity of ad hoc referral.

In the Clinical Prediction Model Testing strategy, it is assumed that an individual general practitioner (GP) would complete the online monogenic diabetes prediction model (http://www.diabetesgenes.org/content/ mody-probability-calculator $)^{9}$ to calculate a probability of the individual having monogenic diabetes (see figure 1). Depending on the probability of the individual having monogenic diabetes as calculated from the prediction 


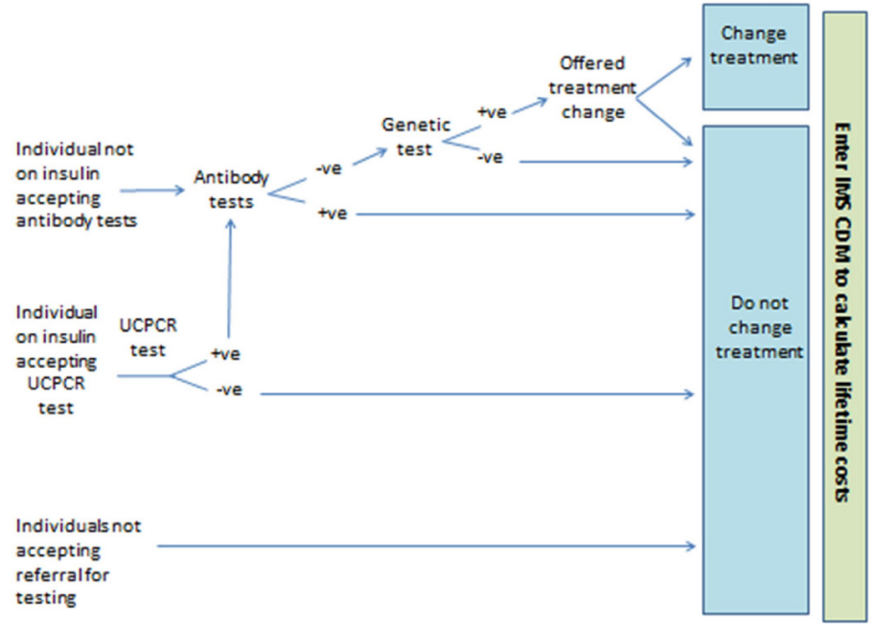

Figure 2 Simplified model structure for the Biomarker Testing strategy. IMS CDM, IMS CORE Diabetes Model; UCPCR, urinary $C$ peptide to creatinine ratio.

model, the GP would then refer them for monogenic diabetes genetic testing or not. Two versions of the prediction model exist, one to distinguish type 1 diabetes from monogenic diabetes (version 1) and the other to distinguish type 2 diabetes from monogenic diabetes (version 2). If the individual is currently receiving insulin, then version 1 of the prediction model is used, otherwise version 2 is used. For each version of the prediction model, nine thresholds are simulated in the decision model. Thus, the Clinical Prediction Model Testing strategy can be evaluated at 81 thresholds (9 from version $1 \times 9$ from version 2 ) for the simulated population. The decision model can then be used to identify the probability threshold for the prediction model that maximises the costs saved using the Clinical Prediction Model Testing strategy compared with the No Testing strategy.

In the Biomarker Testing strategy individuals receive biochemical and/or immunological tests depending on their demonstrated ability to produce insulin (see figure 2). If individuals are currently receiving insulin treatment, they are offered a UCPCR test to determine whether they are producing insulin or not. ${ }^{10}$ Those with a positive UCPCR test are then offered a test for glutamic acid decarboxylase (GAD) and islet antigen 2 (IA2) autoantibodies. ${ }^{11}$ If individuals are not currently receiving insulin treatment it is assumed they can produce their own insulin and so do not require a UCPCR test. Instead, those individuals not on insulin treatment are offered a test for GAD and IA2 autoantibodies. The aim of the GAD and IA2 autoantibodies test is to rule out those individuals with type 1 diabetes who are still producing insulin (ie, in the 'honeymoon' period). Individuals not showing the presence of autoantibodies are then offered the monogenic diabetes genetic test. In the All Testing strategy, all individuals are offered monogenic diabetes genetic testing (see figure 1).
Model input parameters

Population characteristics

The main analysis (modelled Cohort 1 ) simulated a prevalent cohort of individuals in England and Wales who were diagnosed with diabetes when $<30$ years old and were $<50$ years old at the start of the model. The prevalence of monogenic diabetes assumed in this cohort is $2.4 \%$ (GCK mutation $0.7 \%$, HNF1A mutation $1.5 \%$, HNF4A mutation $0.2 \%$ ). A subgroup analysis (modelled Cohort 2) was undertaken to represent a future incident cohort who would have had a diagnosis of diabetes for a shorter duration than those in Cohort 1. Cohort 2 is defined as individuals diagnosed with diabetes when $<30$ years old and who were $<30$ years old at the start of the model, leading to a prevalence of $2.2 \%$ having monogenic diabetes. All information relevant to Cohort 2, including parameter values and results, are in online supplementary data 1 . Further data on the prevalence and characteristics of Cohort 1 are given in online supplementary data 2 .

\section{Test characteristics}

Details of the test sensitivity and specificity used in the model are shown in online supplementary data 3. To calculate the sensitivity and specificity of referral for monogenic diabetes genetic testing in the Ad Hoc Testing strategy, four data sets were used:

- Diabetes prevalence from unpublished data for Tayside, Scotland.

- Estimates of total population by age and area from national census. ${ }^{17}$

- Monogenic diabetes prevalence from the accompanying clinical study. ${ }^{13}$

- Monogenic diabetes genetic test referral rates. ${ }^{7}$

The referral rates for monogenic diabetes genetic testing varied across the UK, with higher referral rates in areas where there is a strong research interest in monogenic diabetes, for example, the south-west of England, and Scotland. Estimates of sensitivity and specificity varied from sensitivity of 0.038 and specificity of 0.996 (Northern Ireland) to sensitivity 0.196 and specificity 0.977 (south-west of England) (see online supplementary data 3). To account for the general low rates of referral in the UK, we assumed the referral rates for one of the lowest areas, northern Ireland. In sensitivity analyses, data from all individual regions were used to estimate sensitivity and specificity for the Ad Hoc Testing strategy. However, the cost of increased awareness in one area compared with other areas is not known, and so it is not possible to estimate the additional cost of increased awareness of monogenic diabetes in the Ad Hoc Testing strategy, such as the south-west of England and Scotland.

For the Clinical Prediction Model Testing strategy the probability thresholds of $10 \%-90 \%$ for the two versions of the test were taken from Shields $e t a l,{ }^{9}$ with sensitivity ranging from 0.5 to 0.99 and specificity ranging from 0.65 to 0.996 . All 81 combinations of probability thresholds were evaluated in the decision model. No adjustments were made to the clinical prediction model as the 
population on which it would be applied (individuals with diabetes in England and Wales) is very similar to that on which it is based. In the Biomarker Testing strategy, sensitivity of 0.94 and specificity of 0.96 for the UCPCR test was used based on a UCPCR cut-off of $\geq 0.2 \mathrm{nmol} /$ mmol to discriminate individuals with $H N F 1 A$ and $H N F 4 A$ mutations who were insulin-treated from individuals with type 1 diabetes. ${ }^{10}$ Besser et al did not report on the sensitivity and specificity of this cut-off to discriminate insulintreated type 2 from GCK, HNF1A and HNF4A mutations, or to discriminate type 1 from GCK mutations. Since use of a different UCPCR cut-off for type 1 or insulin-treated type 2 would be difficult in practice (Besser $e t a l^{10}$ ), we assumed that the UCPCR cut-off of $\geq 0.2 \mathrm{nmol} / \mathrm{mmol}$ could be used to discriminate type 1 from insulin-treated type 2, HNF1A and HNF4A mutations. Furthermore, Besser et al report that UCPCR cannot be used to discriminate GCK from HNF1A and HNF4A mutations. Thus, we assume that the UCPCR cut-off of $\geq 0.2 \mathrm{nmol} / \mathrm{mmol}$ can be used to discriminate type 1 diabetes from insulintreated type 2, GCK, HNF1A and mutations. The impact on the model results of using different estimates of sensitivity and specificity is assessed in sensitivity analyses. Data from McDonald et $a l^{11}$ were used to inform the sensitivity and specificity for the GAD and IA2 autoantibody tests (see online supplementary data 3). For all testing strategies, individuals referred for the monogenic diabetes genetic test were either tested for mutations in the $G C K$ gene only, the $H N F 1 A$ and $H N F 4 A$ genes together, or all three genes (see online supplementary data 2 ).

\section{Uptake and repeat tests}

Using data from the accompanying clinical study, for Cohort 1, it was assumed that $8.2 \%$ of individuals would decline the offer of genetic testing (6.9\% for Cohort 2). This percentage was applied to all of the strategies where genetic testing was an option. For the Biomarker Testing strategy it was assumed that $11.9 \%$ of Cohort $1(12.8 \%$ of Cohort 2) individuals offered the UCPCR test and $8.2 \%$ of Cohort 1 (6.9\% of Cohort 2) individuals offered the autoantibody test would not accept. Estimates of the number of repeat tests required for both cohorts in the Biomarker Testing strategy are reported in online supplementary data 2.

\section{Family genetic testing}

It was assumed in the model that identification of an individual with monogenic diabetes from any of the defined strategies would lead to first degree family members (who fit the defined cohort) also being genetically tested. Once individuals identified from the testing strategies have had the genetic test and are found to have monogenic diabetes, their family members receive the monogenic diabetes genetic tests. In Cohort 1, it was assumed that for every 10 individuals identified by the testing strategies as having monogenic diabetes, a further $6 \cdot 3$ family members are genetically tested, with 5.9 of these assumed to have the mutation (based on UK referral rate data). ${ }^{7}$ These ratios were applied to the Ad Hoc Testing, Clinical Prediction Model Testing and Biomarker Testing strategies.

\section{Treatment for diabetes}

The treatment pattern assumed at the model start is given in online supplementary data 2 . These data are from the accompanying clinical study where the treatment pattern for those truly having monogenic diabetes is based on just 45 individuals. The impact on the model results of the type of treatment at the start of the model is assessed in sensitivity analyses. Only individuals with a positive genetic test were offered a treatment change, which was cessation of diabetes treatment for those with the $G C K$ mutation or to sulphonylureas for individuals with the HNF1A or HNF4A mutations. Data from the clinical study informed the likely treatment pattern once individuals are diagnosed with monogenic diabetes. For Cohort 1, at 1 month after treatment change it was assumed that $86 \%$ of individuals with $H N F 1 A$ or $H N F 4 A$ mutations were receiving a more appropriate treatment, at 3 months this was $86 \%$, at 6 months this was $89 \%$ and at 12 months this was $77 \%$ (see online supplementary data 2). Some individuals having a positive genetic test result may not successfully change to sulphonylurea treatment alone and may continue to receive insulin. ${ }^{18}$ For individuals with $H N F 1 A$ or $H N F 4 A$ mutations it was assumed that they would require insulin treatment eventually, and how much insulin and when they would start taking it would depend on whether they had previously received sulphonylureas and progressed to insulin or had started on insulin initially. As no data are available, two experts in monogenic diabetes $\mathrm{AH}$ and EP) were consulted for their opinion (see online supplementary data 2). Based on data from the accompanying clinical study it was assumed that $93 \%$ of individuals identified to have the GCK mutation, would successfully stop all diabetes treatment.

\section{Resource use}

The type of NHS costs (£), inflated to 2018 prices using the Hospital and Community Health Services pay and prices index ${ }^{19}$ considered within each strategy are summarised in (online supplementary data 4).

All treatment costs were estimated using the reported doses from the clinical study and the British National Formulary. ${ }^{20}$ The costs associated with the tests include costs for the collection of blood and urine samples, costs of the UCPCR and autoantibody tests, and genetic test costs. The costs of nurse time spent providing assistance to those individuals with monogenic diabetes who are changing to a more appropriate treatment were also included (see online supplementary data 4 ) .

The costs associated with home blood glucose monitoring (HBGM) were also included in the model. The frequency of HBGM before and after diagnosis of monogenic diabetes, and any subsequent change in treatment, were estimated from the clinical study for individuals truly having monogenic diabetes (see online supplementary data 2). Data from the literature were used to inform 
HBGM frequency in individuals with type 1 and type 2 diabetes. ${ }^{21}{ }^{22}$ It was assumed that individuals who have a GCK, HNF1A or HNF4A mutation, but did not have a genetic test or change treatment would have the same HBGM frequency as at the start of the model. Costs of HBGM were based on use of the Accu-Check Aviva metre (£16.09 for 50 strips) ${ }^{20}$

The costs of diabetes-related complications for individuals with type 1 diabetes, type 2 diabetes, and $H N F 1 A$ or HNF4A mutations were identified from reviewing the published literature and using data from the National Schedule of Reference Costs 2016/2017. Only cost data from the UK were modelled in the IMS CDM (see online supplementary data 4). The majority of cost estimates from the literature were associated with uncertainty, mainly in inflating the costs to 2018 due to the age of the evidence available, therefore all of the long-term costs inputted into the model were rounded to the nearest $£ 50$ to avoid spurious precision. It is assumed that individuals with $G C K$ mutations do not experience long-term diabetes-related complications ${ }^{3}$ and once identified as having a mutation in the GCK gene, they no longer incur the costs of diabetes-specific consultations. Data from Curtis $2017^{19}$ and Currie et al $2010^{23}$ were used to inform the costs of diabetes-specific consultations (see online supplementary data 4 ).

\section{Long-term events and survival}

It was assumed that individuals with $G C K$ mutations do not experience diabetes-related events and have the same mortality rate as the general population. ${ }^{17}$ Therefore inidviduals with GCK mutations do not enter the IMS CDM. For individuals with $H N F 1 A$ and $H N F 4 A$ mutations, due to limited data on long-term complications and mortality, it was assumed that these individuals have the same pattern of long-term complications and mortality as individuals with type 1 diabetes. Therefore individuals with $H N F 1 A$ and HNF4A mutations were modelled using the type 1 diabetes model in the IMS CDM.

\section{Model outcomes}

All costs (£, 2018) beyond the first year are discounted at a rate of $3.5 \%$ per annum to account for the preference for deferring future costs in economic evaluations. ${ }^{24}$ Discounted and undiscounted total costs are reported in the results section alongside the estimated discounted incremental costs per person with diabetes over a lifetime for each strategy compared with the No Testing strategy and the proportion of monogenic diabetes cases identified by each strategy.

\section{Analysis}

The results of a 'base case' analysis are presented, but due to the uncertainty surrounding many of the parameter estimates, alternative combinations of assumptions may be equally plausible. Therefore, wide-ranging one-way sensitivity and threshold analyses have been conducted to explore the different sources of uncertainty; this includes an analysis where an improvement in utility for those who successfully change treatment is assumed. Details of the sensitivity and threshold analyses undertaken for Cohort 1 can be found in online supplementary data 2 (see online supplementary data 1 for details on Cohort 2 analyses). In contrast to our planned analysis, ${ }^{12}$ we decided not to do a probabilistic analysis because important structural uncertainties in this model could not be fully captured by a probabilistic analysis (it would therefore be misleading).

\section{Patient and public involvement}

There was no patient and public involvement in the development or analysis of the model.

\section{RESULTS}

Cohort 1: diagnosed $<30$ years old, $<50$ years old at start of model

For the 'base case' analysis, the total discounted costs per person with diabetes over a lifetime were estimated to be $£ 53600-£ 54000$ depending on the strategy used (see table 1). The All Testing strategy was estimated as

Table 1 Summary of the per person lifetime costs ${ }^{\star}$ and percentage of cases and non-cases genetically tested for each strategy (ordered by increasing cost of strategy)

\begin{tabular}{|c|c|c|c|c|c|}
\hline \multirow[b]{2}{*}{ Strategy } & \multirow[b]{2}{*}{$\begin{array}{l}\text { Total undiscounted } \\
\text { costs* }\end{array}$} & \multirow[b]{2}{*}{$\begin{array}{l}\text { Total discounted } \\
\text { costs* }^{\star}\end{array}$} & \multirow{2}{*}{$\begin{array}{l}\text { Incremental costs } \\
\text { versus no testing } \\
\text { strategy* }\end{array}$} & \multicolumn{2}{|c|}{$\%$ who are genetically tested } \\
\hline & & & & $\begin{array}{l}\text { With monogenic } \\
\text { diabetes }\end{array}$ & $\begin{array}{l}\text { Without monogenic } \\
\text { diabetes }\end{array}$ \\
\hline $\begin{array}{l}\text { Clinical Prediction } \\
\text { Model Testingt }\end{array}$ & $£ 133200$ & $£ 53600$ & $-£ 100$ & 92 & 3 \\
\hline Biomarker Testing & $£ 133300$ & $£ 53600$ & $-£ 100$ & 92 & 8 \\
\hline Ad Hoc Testing & $£ 133500$ & $£ 53700$ & 0 & 6 & $<1$ \\
\hline No Testing & $£ 133600$ & $£ 53700$ & NA & 0 & 0 \\
\hline All Testing & $£ 133700$ & $£ 54000$ & $£ 300$ & 92 & 92 \\
\hline
\end{tabular}

${ }^{*}$ Rounded to nearest $£ 100$.

†Probability thresholds chosen to maximise costs saved versus No Testing are $12.6 \%$ for type 1 versus monogenic diabetes and $75.5 \%$ for type 2 versus monogenic diabetes. 
the most costly $(£ 54,000)$, the cheapest options were the Clinical Prediction Model Testing (where the probability thresholds were chosen to maximise costs saved compared with No Testing) and Biomarker Testing strategies $(£ 53,600)$. The No Testing and Ad Hoc Testing strategies were both estimated as $£ 53700$ per person with diabetes over a lifetime. The Ad Hoc Testing strategy was estimated to identify very few cases of monogenic diabetes (6\%) compared with the All Testing strategy which was estimated to identify $92 \%$ of monogenic diabetes cases. No more than $92 \%$ of monogenic diabetes cases can be identified by any strategy due to the assumption that $8 \%$ of individuals will not accept an offer of genetic testing for monogenic diabetes. Family testing boosts the detection of monogenic diabetes cases to $92 \%$ in the Clinical Prediction Model Testing and Biomarker Testing strategies. The costs saved for these two strategies over the No Testing strategy relate to more individuals getting a monogenic diabetes diagnosis and changing to receive more appropriate treatment which is cheaper and also leads to a reduction in the frequency of HBGM. The All Testing strategy is the most expensive since although more monogenic diabetes diagnoses are made, resulting in fewer treatment and HBGM costs, the costs of genetically testing all individuals diagnosed with diabetes are very high.

As there are 81 different combinations of probability thresholds for the clinical prediction model, the combination of thresholds which maximises the costs saved for the Clinical Prediction Model Testing strategy have been reported above. In figure 3, all 81 threshold combinations for the clinical prediction model are shown. The Clinical Prediction Model Testing strategy is estimated to identify $74 \%-92 \%$ of monogenic diabetes cases depending on the probability threshold combinations used to refer individuals for genetic testing. The lifetime costs saved per person with these threshold combinations compared with No Testing vary from $£ 0$ to $£ 150$.

Sensitivity analysis results suggest that the impacts on costs in the different scenarios are insensitive to wideranging, plausible changes to key model parameters, (see

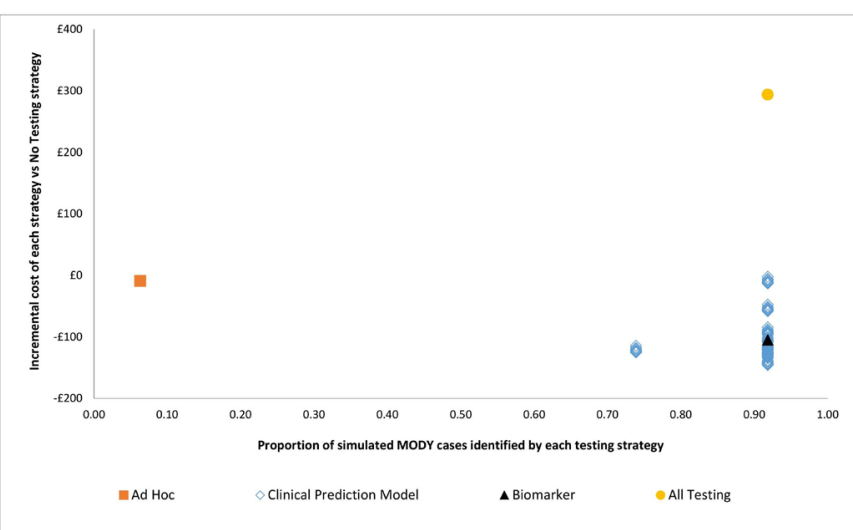

Figure 3 Base case incremental costs (vs No Testing) and the proportion of monogenic diabetes cases identified for each strategy.

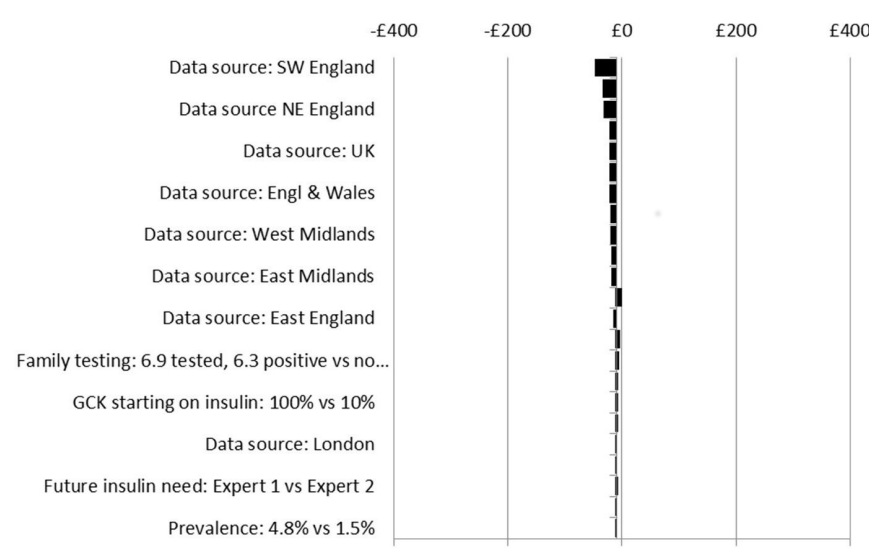

Figure 4 Sensitivity analyses: incremental costs per person over a lifetime for the Ad Hoc Testing strategy versus the No Testing strategy. GCK, glucokinase.

figures 4-7). No plausible parameter value changes the finding that the Ad Hoc Testing and Clinical Prediction Model Testing strategies are always estimated to save costs compared with the No Testing strategy. Only extreme assumptions on the uptake of genetic and UCPCR testing (just 10\% uptake) suggest fewer costs are saved from the Biomarker Testing strategy when compared with the No Testing strategy. Except for assumptions on test uptake, the estimated cost savings are in the region of $£ 0-£ 50$ per person over a lifetime for the Ad Hoc Testing strategy (see figure 4), £50-£300 for the Clinical Prediction Model Testing strategy (see figure 5) and £50-£250 for the Biomarker Testing strategy (see figure 6). The All Testing strategy is estimated to cost an additional £150$£ 350$ per person over a lifetime compared with the No Testing strategy except when the cost of the genetic test is assumed to be $<60 \%$ of its current cost (see figure 7 ).

As figures 4-7 show, the findings are most sensitive to:

- The estimated prevalence of monogenic diabetes within the cohort-increasing prevalence (from $2.4 \%$ to $4.8 \%$ ) leads to greater costs saved for the Ad

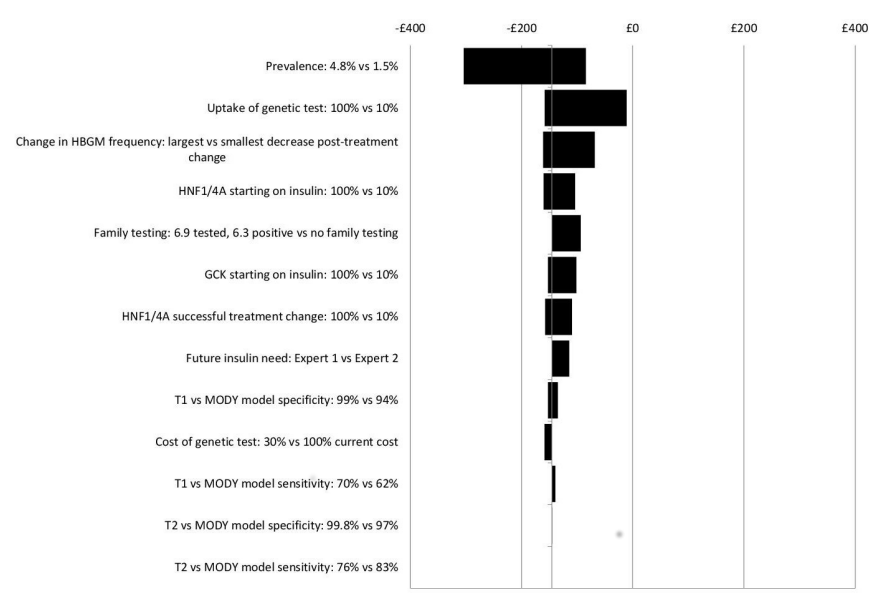

Figure 5 Sensitivity analyses: incremental costs per person over a lifetime for the Clinical Prediction Model Testing strategy versus the No Testing strategy. GCK, glucokinase; HBGM, home blood glucose monitoring; HNF1/4A, hepatocyte nuclear factor 1/4 alpha. 


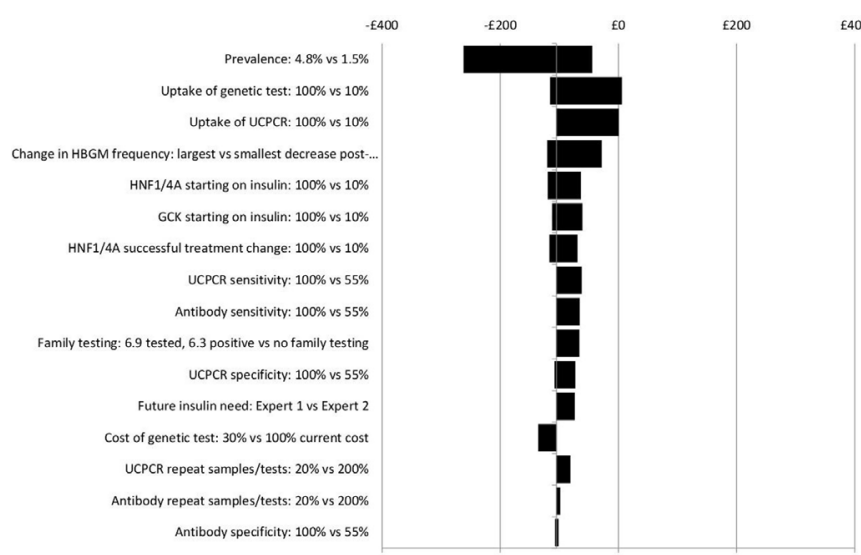

Figure 6 Sensitivity analyses: incremental costs per person over a lifetime for the Biomarker Testing strategy vs the No Testing strategy. GCK, glucokinase; HBGM, home blood glucose monitoring; HNF1/4A, hepatocyte nuclear factor 1/4 alpha; UCPCR, urinary $C$ peptide to creatinine ratio.

Hoc Testing, Clinical Prediction Model Testing and Biomarker Testing strategies compared with the No Testing strategy.

- The uptake of testing-reduced uptake leads to fewer costs saved for all strategies compared with the No Testing strategy,

- The frequency of HBGM pretreatment and posttreatment change-assuming that individuals change their frequency of HBGM by only a small amount after a diagnosis of monogenic diabetes, leads to fewer costs saved compared with the No Testing strategy,

- The proportion of individuals with monogenic diabetes who receive insulin before their monogenic diabetes diagnosis-the larger the proportion receiving insulin before being diagnosed as having monogenic diabetes, the greater the costs saved for all strategies compared with No Testing.

Threshold analysis results (see online supplementary data 2) suggest that when the genetic tests are reduced to

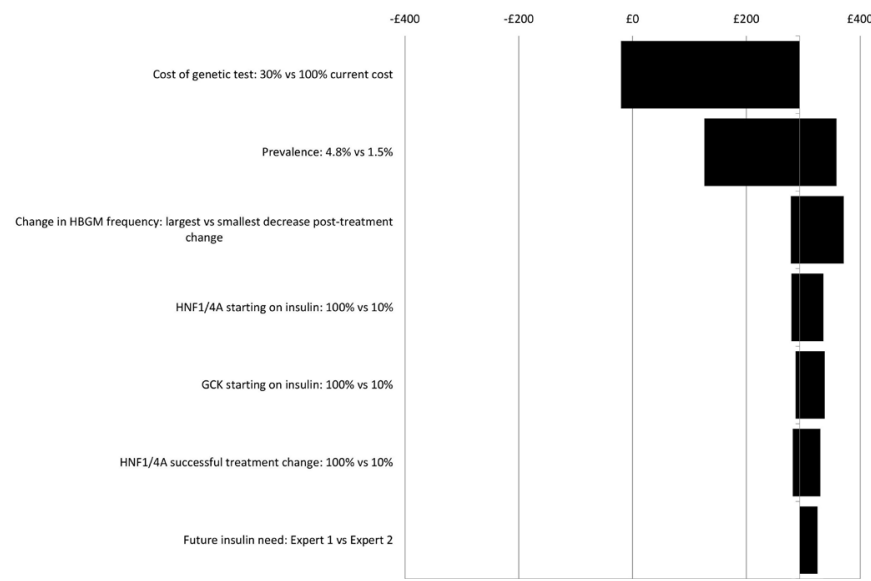

Figure 7 Sensitivity analyses: incremental costs per person over a lifetime for the All Testing strategy versus the No Testing strategy. GCK, glucokinase; HBGM, home blood glucose monitoring; HNF1/4A, hepatocyte nuclear factor 1/4 alpha. approximately $35 \%$ of their current costs, the All Testing strategy incurs no additional costs compared with the No Testing strategy. However, in this situation, the Biomarker Testing and Clinical Prediction Model Testing strategies are estimated to save, approximately $£ 150$ per person over a lifetime, compared with the No Testing strategy. Reducing the percentage of individuals with monogenic diabetes who are receiving only insulin at the start of the model has little impact on the incremental costs estimated: even if $10 \%$ of individuals with GCK mutations or $10 \%$ of individuals with $H N F 1 A$ or $H N F 4 A$ mutations are on tablets at the start of the model, slight cost savings are still estimated with the Clinical Prediction Model Testing and Biomarker Testing strategies compared with the No Testing strategy (see figures 5 and 6).

Threshold analyses specific to the Biomarker Testing strategy demonstrate that once uptake of the UCPCR and autoantibody tests is reduced to less than $70 \%$, the costs saved with the Biomarker Testing strategy compared with the No Testing strategy reduce. Costs saved with the Biomarker Testing strategy are most sensitive to reductions in the sensitivity of the UCPCR and autoantibody tests. Increases in the number of repeat urine or blood samples and tests required within the Biomarker Testing strategy have little impact on the estimate of costs saved compared with the No Testing strategy.

\section{Cohort 2: diagnosed $<30$ years, $<30$ years at start of model}

As in Cohort 1, the Clinical Prediction Model Testing and Biomarker Testing strategies are estimated to save $£ 100$ per person with diabetes over a lifetime compared with the No Testing strategy, while the All Testing strategy is assumed to cost an additional $£ 300$ compared with the No Testing strategy. When compared with Cohort 1, the Clinical Prediction Model Testing and Biomarker Testing strategies are not estimated to save any more costs because of the trade-off between individuals being less likely to be on insulin prior to genetic testing in Cohort 2 (67\% vs 83\% in Cohort 1) even though they are more likely to successfully change to sulphonylureas than Cohort 1 (100\% vs $79 \%$ in Cohort 1). Individuals in Cohort 2 were estimated to monitor their blood glucose less frequently before receiving a diagnosis of monogenic diabetes compared with Cohort 1, and so fewer costs are saved from reducing further the HBGM frequency than is the case for Cohort 1. See online supplementary data 1 for further results, including sensitivity analyses which suggest that estimates of prevalence and testing uptake have the largest impact on the findings (as for Cohort 1).

\section{DISCUSSION}

The Clinical Prediction Model Testing and Biomarker Testing strategies modelled here have been estimated to be cost saving for identifying individuals with monogenic diabetes and changing their treatment compared with the current practice of no genetic testing. Assumptions about the prevalence of monogenic diabetes within the 
simulated cohort, the uptake of testing and the frequency of HBGM before and after receiving a diagnosis of monogenic diabetes had the largest impact on the findings, but did not change the overall conclusions that targeted strategies are estimated to save costs compared with the No Testing or All Testing strategies. Data on prevalence and test uptake were taken directly from the accompanying clinical study, which is the first to systematically estimate prevalence of monogenic diabetes in the UK. ${ }^{13}$ Information on the frequency of HBGM before and after a diagnosis of monogenic diabetes is based on just a small number of individuals, but is currently the best evidence available.

This is the first UK-based economic evaluation of strategies to identify individuals with monogenic diabetes. A published paper documented the development of the model and the intended analysis, ${ }^{12}$ and the minor departures from the protocol have been declared and justified. UK data have been used to inform many of the model inputs for which there was previously no credible evidence. However, due to the rarity of monogenic diabetes, many inputs specific to individuals with monogenic diabetes are based on very few individuals, especially for Cohort 2 , or assumptions. For instance, it was assumed that treatment and HBGM frequency data taken from the clinical study at 12 months follow-up remained constant over time in the model, with additional long-term treatment data informed by clinical opinion. Until longer follow-up data are available, it is unclear what impact these assumptions may have on the model results.

We simulated two cohorts, both based on data from the clinical study. The aim of Cohort 2 was to assess the impact of strategies for identifying monogenic diabetes in individuals more recently diagnosed with diabetes than those in Cohort 1. Although it was anticipated that individuals in Cohort 2 would find it easier to change to more appropriate treatment (because they had not been on their existing treatment for a long time), we actually found that individuals in Cohort 2 were less likely to be on insulin at that point, so costs saved from changing treatment were smaller than for Cohort 1 , even though more individuals changed treatment. However this analysis was limited by the low number of participants close to diagnosis for which data were available. Furthermore, the performance of the Clinical Prediction Model Testing and Biomarker Testing strategies are based on prevalent cohorts $^{9-11}$ which will impact on their generalisability to an incident cohort (Cohort 2). Thus, there are still many uncertainties associated with the results, including that the IMS CDM has not been validated for monogenic diabetes, so these results should be interpreted with this in mind. Nevertheless, the numerous sensitivity and threshold analyses estimated cost savings for the Clinical Prediction Model Testing (when choice of thresholds was maximised to save costs) and Biomarker Testing strategies compared with No Testing.

Naylor et $a l^{25}$ conducted an economic evaluation of genetic testing (akin to our All Testing strategy) for monogenic diabetes in individuals aged 25-40 years who were newly diagnosed with type 2 diabetes compared with no genetic testing from a US health system perspective. Individuals identified as having $H N F 1 A$ or $H N F 4 A$ mutations who successfully transferred to sulphonylureas were assumed a HbAlc reduction of $16.4 \mathrm{mmol} / \mathrm{mol}$ compared with those not changing treatment (based on six individuals at 3 months follow-up after treatment change ${ }^{26}$ and a utility increase of 0.13 for transferring from insulin to sulphonylurea treatment (based on evidence from 519 individuals aged 65 years and older with type 2 diabetes). ${ }^{27}$ Naylor et al reported a gain of $0 \cdot 012$ quality-adjusted lifeyears (QALYs) for the testing strategy at an additional cost of $\$ 2400$ per person over a lifetime compared with their no testing strategy, resulting in an incremental costeffectiveness ratio of $\$ 205000$ per QALY gained. ${ }^{25}$ The additional costs for the genetic testing strategy in Naylor et $a l^{25}$ are much greater than the All Testing strategy in our evaluation ( $\$ 2400$ vs $£ 300$ ) because of differences in the populations simulated. In our evaluation a younger diabetes population is assumed, with individuals who truly have monogenic diabetes being more likely to be misdiagnosed with type 1 and receive insulin. The simulated population in Naylor et al is older and explicitly those diagnosed with type 2 , therefore are less likely to receive insulin treatment, so have fewer cost savings from changing treatment.

The health impacts assumed by Naylor et $a l^{25}$ are also different from those observed in our accompanying clinical study. Using the EQ-5D Index, we found little evidence over the 12 months treatment change period for an improvement in utility associated with more appropriate treatment, although the EQ-5D visual analogue scale and the Diabetes Treatment Satisfaction Questionnaire did suggest an improvement at 12 months. Furthermore, in the sample of 28 individuals with $H N F 1 A$ or $H N F 4 A$ mutations who successfully changed to sulphonylureas, no statistically significant impact on HbAlc at 12 months after treatment change was found (mean difference of $3.43 \mathrm{mmol} / \mathrm{mol}(95 \%$ CI -2.18 to 9.04$)$ ). Due to the lack of evidence suggesting an effect on quality of life and HbAlc we took the decision to assume there were no differences in quality of life and $\mathrm{HbAlc}$ between those identified as having monogenic diabetes and subsequently changing treatment, and those not identified. Our evaluation was conservative, as evidence shows that changing treatment can have a substantial beneficial impact on individuals. ${ }^{28} 29$ A sensitivity analysis assuming an improvement in utility for those found to have $H N F 1 A$ or HNF4A mutations who successfully changed treatment indicated $<5$ quality-adjusted days were gained from the Clinical Prediction Model, Biomarker and All Testing strategies compared with No Testing. However, generic and relatively simple quality of life measures (eg, EQ-5D) are likely to be insensitive to the magnitude and type of changes individuals with diabetes might experience when changing to more appropriate treatment. Measuring such changes to quality of life is also limited by the ceiling 
effect, since these individuals generally constitute a wellcontrolled, young diabetes population with a good quality of life. Given these limitations we have not considered any reductions in quality of life that may occur during the testing period, especially for those tested but not found to have monogenic diabetes.

A further limitation is in the evidence used to inform the sensitivity and specificity of the testing strategies. For example, the accuracy of antibody testing for the Biomarker strategy is based on a two-gate study design where the test is evaluated by comparing test results in individuals known to have a diagnosis of monogenic diabetes with those newly diagnosed with type 1 diabetes. Such study designs have been shown to lead to overstated accuracy estimates. ${ }^{30}$

A limitation of the Ad Hoc Testing strategy is in choosing the referral rates that are representative. We used referral rates for the area with the lowest rate of referral. We could have used an average referral rate across the country, but would not have been able to capture the relevant costs of the increased awareness in some areas (such as the southwest of the UK where the referral centre for monogenic diabetes is based) which is linked to increased referral.

The results suggest that within the context of the NHS, the additional costs of genetically testing (a relatively large number of) individuals are likely to be offset by the lifetime savings from the subsequent treatment changes in a very small proportion of individuals. Although the estimated cost savings are relatively small per person (approximately £100-£200 over a lifetime), assuming there are approximately 200000 individuals (personal communication) in England and Wales who are $<50$ years old and have had a diagnosis of diabetes before the age of 30 years, between $£ 20$ million and $£ 40$ million could be saved if such strategies are used. To be able to apply these findings to other populations the cost of the testing in particular will need to be updated. If the genetic test costs are significantly higher, then it is unclear whether the Clinical Prediction Model Testing and Biomarker Testing strategies could be considered cost saving, or even cost neutral. However, further collection of treatment pattern, HBGM frequency, HbAlc and quality of life data for individuals with monogenic diabetes is required to better inform the decision model, especially to model an incident cohort. Additional strategies to better identify those with monogenic diabetes are feasible, and in development, but will also require evaluation for their effectiveness and cost-effectiveness.

\section{CONCLUSIONS}

Targeted strategies to identify individuals with monogenic diabetes and change to more appropriate treatment may be cost saving to the NHS. However, collection of longerterm treatment and frequency of HBGM data would be valuable to reduce the main uncertainties in the modelling. Future work to evaluate the use of genetic testing strategies soon after diagnosis of diabetes would be useful to policy makers.

Twitter Rob Anderson @RobAinHSR and Maggie Shepherd @MaggieShepher13 Acknowledgements The authors thank IMS Health for use of the IMS CDM.

Contributors JLP designed the decision model, contributed to data collection, undertook analysis and interpretation of the model results and drafted the manuscript. RA and $\mathrm{CH}$ helped design and analyse the decision model, and contributed to the interpretation of the results and drafting of the manuscript. BS, $\mathrm{MH}, \mathrm{MS}$, TJM, EP and AH contributed to the study design and data collection, and commented on the manuscript. SK contributed to data collection and commented on the manuscript.

Funding This study was supported by the Department of Health and Wellcome Trust Health Innovation Challenge Award (HICF-1009-041 and WT-091985). JLP is partly supported by the NIHR Collaboration for Leadership in Applied Health Research and Care for the South West Peninsula (PenCLAHRC). BS, MH and AH are core members of the National Institute for Health Research (NIHR) Exeter Clinical Research Facility. TJM is supported by an NIHR Chief Scientist Office Fellowship. $\mathrm{AH}$ is a Wellcome Trust Senior Investigator and an NIHR Senior Investigator. EP is a Wellcome Trust New Investigator. MS is a National Institute for Health Research (NIHR) Senior Nurse and Midwife Research Leader (NIHR4-SNMRL058) and is also supported by the Exeter NIHR Clinical Research Facility at the University of Exeter.

Competing interests None declared.

Patient consent for publication Not required.

Provenance and peer review Not commissioned; externally peer reviewed.

Data availability statement No data are available. The decision analytic model described in this manuscript is not available due to the IMS CDM being under license for the current study.

Open access This is an open access article distributed in accordance with the Creative Commons Attribution 4.0 Unported (CC BY 4.0) license, which permits others to copy, redistribute, remix, transform and build upon this work for any purpose, provided the original work is properly cited, a link to the licence is given, and indication of whether changes were made. See: https://creativecommons.org/ licenses/by/4.0/.

Author note Sophie King is currently at Centre for Trials Research, Cardiff University, Cardiff, UK

\section{ORCID iDs}

Jaime L Peters http://orcid.org/0000-0003-1778-3518

Michelle Hudson http://orcid.org/0000-0003-0623-1873

Maggie Shepherd http://orcid.org/0000-0003-2660-0955

\section{REFERENCES}

1 Tattersall RB. Mild familial diabetes with dominant inheritance. Q J Med 1974;43:339-57.

2 Murphy R, Ellard S, Hattersley AT. Clinical implications of a molecular genetic classification of monogenic beta-cell diabetes. Nat Clin Pract Endocrinol Metab 2008;4:200-13.

3 Steele AM, Shields BM, Wensley KJ, et al. Prevalence of vascular complications among patients with glucokinase mutations and prolonged, mild hyperglycemia. JAMA 2014;311:279-86.

4 Stride A, Shields B, Gill-Carey O, et al. Cross-Sectional and longitudinal studies suggest pharmacological treatment used in patients with glucokinase mutations does not alter glycaemia. Diabetologia 2014;57:54-6.

5 Pearson ER, Starkey BJ, Powell RJ, et al. Genetic cause of hyperglycaemia and response to treatment in diabetes. Lancet 2003;362:1275-81.

6 Thanabalasingham G, Owen KR. Diagnosis and management of maturity onset diabetes of the young (MODY). BMJ 2011;343:d6044.

7 Shields BM, Hicks S, Shepherd MH, et al. Maturity-Onset diabetes of the young (MODY): how many cases are we missing? Diabetologia 2010;53:2504-8.

8 Fajans SS, Bell GI, Bowden DW, et al. Maturity-Onset diabetes of the young. Life Sci 1994;55:413-22.

9 Shields BM, McDonald TJ, Ellard S, et al. The development and validation of a clinical prediction model to determine the probability of MODY in patients with young-onset diabetes. Diabetologia 2012;55:1265-72. 
10 Besser REJ, Shepherd MH, McDonald TJ, et al. Urinary C-peptide creatinine ratio is a practical outpatient tool for identifying hepatocyte nuclear factor 1-\{alpha\}/hepatocyte nuclear factor 4-\{alpha\} maturityonset diabetes of the young from long-duration type 1 diabetes. Diabetes Care 2011;34:286-91.

11 McDonald TJ, Colclough K, Brown R, et al. Islet autoantibodies can discriminate maturity-onset diabetes of the young (MODY) from type 1 diabetes. Diabet Med 2011;28:1028-33.

12 Peters JL, Anderson R, Hyde C. Development of an economic evaluation of diagnostic strategies: the case of monogenic diabetes. BMJ Open 2013;3. doi:10.1136/bmjopen-2013-002905. [Epub ahead of print: 28 May 2013]

13 Shields BM, Shepherd M, Hudson M, et al. Population-Based assessment of a biomarker-based screening pathway to aid diagnosis of monogenic diabetes in young-onset patients. Diabetes Care 2017;40:1017-25.

14 EuroQol Group. EuroQol--a new facility for the measurement of health-related quality of life. Health Policy 1990;16:199-208.

15 Husereau D, Drummond M, Petrou S, et al. Consolidated health economic evaluation reporting standards (cheers) statement. Pharmacoeconomics 2013;31:361-7.

16 Palmer AJ, Roze S, Valentine WJ, et al. The core diabetes model: projecting long-term clinical outcomes, costs and cost-effectiveness of interventions in diabetes mellitus (types 1 and 2) to support clinical and reimbursement decision-making. Curr Med Res Opin 2004;20 Suppl 1:S5-26.

17 Office for National Statistics. Population estimates by ethnic group 2002-2009 Statistical Bulletin; 2011.

18 Shepherd M, Shields B, Ellard S, et al. A genetic diagnosis of HNF1A diabetes alters treatment and improves glycaemic control in the majority of insulin-treated patients. Diabet Med 2009;26:437-41.

19 Curtis LA, Burns A. Unit costs of health and social care 2017: personal social services research unit. University of Kent, 2017.
20 National Institute for Health and Clinical Excellence. BNF; 2018

21 Farmer AJ, Wade AN, French DP, et al. Blood glucose self-monitoring in type 2 diabetes: a randomised controlled trial. Health Technol Assess 2009;13:1-50.

22 Yeaw J, Lee WC, Aagren M, et al. Cost of self-monitoring of blood glucose in the United States among patients on an insulin regimen for diabetes. J Manag Care Pharm 2012;18:21-32.

23 Currie CJ, Gale EAM, Poole CD. Estimation of primary care treatment costs and treatment efficacy for people with type 1 and type 2 diabetes in the United Kingdom from 1997 to $2007^{\star}$. Diabet Med 2010;27:938-48.

24 National Institute for Health and Clinical Excellence. Guide to the methods of technology appraisal; 2008.

25 Naylor RN, John PM, Winn AN, et al. Cost-Effectiveness of MODY genetic testing: translating genomic advances into practical health applications. Diabetes Care 2014;37:202-9.

26 Thanabalasingham G, Pal A, Selwood MP, et al. Systematic assessment of etiology in adults with a clinical diagnosis of young-onset type 2 diabetes is a successful strategy for identifying maturity-onset diabetes of the young. Diabetes Care 2012;35:1206-12.

27 Huang ES, Shook M, Jin L, et al. The impact of patient preferences on the cost-effectiveness of intensive glucose control in older patients with new-onset diabetes. Diabetes Care 2006;29:259-64.

28 Shepherd M, Hattersley AT. 'I don't feel like a diabetic any more': the impact of stopping insulin in patients with maturity onset diabetes of the young following genetic testing. Clin Med 2004;4:144-7.

29 Shepherd M, Miles S, Jones J, et al. Differential diagnosis: identifying people with monogenic diabetes. J Diabetes Nurs 2010;14:342-7.

30 Rutjes AWS, Reitsma JB, Vandenbroucke JP, et al. Case-Control and two-gate designs in diagnostic accuracy studies. Clin Chem 2005;51:1335-41. 